\title{
A European survey on the detection and management of iron overload in transfusion-dependent patients with MDS
}

\author{
Aristoteles Giagounidis • Susanna Leto di Priolo • \\ Susanne Ille $\cdot$ Pierre Fenaux
}

Received: 29 July 2010 / Accepted: 23 December 2010 / Published online: 16 February 2011

(C) The Author(s) 2011. This article is published with open access at Springerlink.com

\begin{abstract}
To better understand the detection and management of iron overload in transfusion-dependent patients with myelodysplastic syndromes (MDS), a 15-min web- or paper-based survey was conducted among 338 European physicians from 27 countries. Respondents had a mean of 18 years of clinical experience. Forty-six percent and 27\% of physicians noted that detecting and treating iron overload were either "very important" or "important," respectively. The main reason for not actively exploring iron overload was related to poor patient prognosis, while the main reasons for not initiating iron chelation therapy were poor patient prognosis and older patient age. Thirty-seven percent and $31 \%$ of physicians believed that treating iron overload in these patients was "very important" or "important," respectively. Ninety percent of physicians prescribed iron chelation therapy, and $38 \%$ of transfusion-
\end{abstract}

\author{
A. Giagounidis $(\bowtie)$ \\ Medizinische Klinik II, St. Johannes Hospital, \\ An der Abtei 7-11, \\ 47166 Duisburg, Germany \\ e-mail: gia@krebs-duisburg.de \\ S. Leto di Priolo \\ Novartis Farma SpA, \\ Origgio, Italy \\ S. Ille \\ HealthCare Division, GfK SE, \\ Nuremberg, Germany \\ P. Fenaux \\ European School of Haematology, Hôpital Saint-Louis, \\ Paris, France \\ P. Fenaux \\ Service d'hématologie clinique, \\ Hôpital Avicenne/Université Paris, \\ Paris, France
}

dependent patients received iron chelation therapy. The key reasons for not initiating iron chelation therapy were related to poor patient prognosis ( $72 \%$ ), patient age $\geq 85$ years $(50 \%)$, and comorbidities $(34 \%)$. The views of these experienced MDS physicians reflect available international MDS treatment guidelines.

Keywords MIDIS · Myelodysplastic syndromes . Transfusions · Iron overload · Iron chelation · Survey

\section{Introduction}

Red blood cell (RBC) transfusions are a common therapy to treat symptomatic anemia that affects most patients with myelodysplastic syndromes (MDS) [1]. Several retrospective studies have shown that patients who become RBC transfusion-dependent have a significantly shorter overall survival than those who are not dependent on transfusions $[2,3]$. This decreased survival may in part be due to patients accumulating excess iron and/or to intrinsically more severe bone marrow disease than in nondependent patients. International Prognostic Scoring System (IPSS) Low- and Intermediate-1 (Int-1)-risk patients, who may have prolonged survival, receive blood transfusions for many years and may be exposed to a greater risk of iron overload, leading to progressive dysfunction in organs such as the liver, heart, and endocrine glands [2, 4].

Guidelines from Spain [5], the Italian Society of Hematology [6], the UK MDS Guidelines Group [7], the National Comprehensive Cancer Network in the USA [8], and the MDS Foundation [4], all recommend the use of iron chelation therapy in patients with Low- or Int-1-risk MDS who have received more than 20 to 50 transfusions. In Europe, these recommendations, although not based on 
controlled clinical trials demonstrating superiority of iron chelation versus observation alone, have an impact on the perceptions and prescriptions of iron chelation therapy in patients with MDS. There is currently some controversy regarding the use of iron chelation therapy in these patients, particularly because they have a median age of 70 years, and many patients with a poor prognosis may not survive long enough to develop the clinical consequences of iron overload [9-11]. There is some argument, however, that even patients with higher-risk MDS may also benefit from iron chelation therapy through improved outcome of allogeneic stem cell transplantation, possible decreases in infection, and although controversial, delayed leukemic transformation in some cases [6, 12].

In order to understand what physicians treating transfusion-dependent patients with MDS in Europe believe to be the reasons for their willingness or reservations toward detecting and managing iron overload, a survey of European physicians has been conducted. The MDS IronOverload Detection Insight Survey (MIDIS) was conducted by the MDS Foundation and the European School of Haematology (ESH) in partnership with Novartis Oncology. The results of the survey are presented here.

\section{Design and methods}

\section{Inclusion/exclusion criteria}

To participate in the study, respondents had to be physicians from a European country, either fully qualified, in training or completing their internship, and seeing at least one transfusion-dependent patient with MDS in an average 6month period.

MIDIS was initiated at the 2008 meeting of the American Society of Hematology and was also distributed at other international conferences via e-mails, letters, and flyers, as well as links to the survey hosted on the MDS Foundation and ESH websites.

\section{Study questionnaire}

MIDIS was a quantitative survey that comprised up to 33 structured questions that could be completed either on paper or online in approximately $15 \mathrm{~min}$. The questions were reviewed by a study steering committee, comprising the authors and external experts, prior to being included in the survey. The questions were related to profiling and demographics of the respondents (qualification status, primary specialty, work setting, number of transfusiondependent patients with MDS seen, and involvement in MDS), detection of iron overload (frequency of serum ferritin testing, influence of serum ferritin tests on treatment decisions, importance of detecting iron overload, and potential reasons for and reservations against detection), and treatment of iron overload with chelation therapy (importance of treatment, use of iron chelation therapy, markers for therapy initiation, number of patients who receive iron chelation therapy, and potential reasons for and reservations against iron chelation therapy). Questions relating to detection and treatment of iron overload were answered using a scale from 1 (indicating not important at all) to 7 (very important). Questions relating to barriers and triggers for detecting and treating iron overload were also answered using a scale from 1 (indicating not a barrier or trigger at all) to 7 (a strong barrier or trigger).

Analysis

Collection and analysis of the MIDIS results were undertaken by the HealthCare Division of GfK SE. The results for both respondents' background, and barriers and triggers toward detection and treatment of iron overload, were analyzed quantitatively.

\section{Results}

Respondent demographics

A total of 338 physicians from 27 European countries participated in the survey (Table 1). Their profile shows that they were experienced clinicians specializing mostly in hematology (Table 2). A total of $88 \%$ were involved in diagnosing patients with MDS, $87 \%$ initiated treatment for MDS, and the same proportion was involved in the maintenance of treatment for MDS. The survey was completed by the respondents themselves.

\section{Detection of iron overload}

On a scale from 1 (not important at all) to 7 (very important), the majority of the respondents believed that detection of iron overload in transfusion-dependent patients with MDS was "very important" (score 7; 46\% of respondents) or "important" (score 6; 27\%; Fig. 1). The mean score for the subjective importance of detecting iron overload in transfusion-dependent patients with MDS was 6.1 .

On a similar 1-7 scale, most respondents believed that it was "very likely" (score 7; 40\%) or "likely" (score 6; 25\%) that iron overload would be detected at their institution (mean score $=5.8$ ). Approximately half of the respondents $(53 \%)$ said that they monitored serum ferritin levels in their transfusion-dependent patients every 3 months, with $26 \%$ responding that the frequency of serum ferritin monitoring 
Table 1 Countries represented in the survey

\begin{tabular}{|c|c|}
\hline Country & Responses \\
\hline Austria & 8 \\
\hline Belgium & 22 \\
\hline Bulgaria & 4 \\
\hline Croatia & 4 \\
\hline Czech Republic & 19 \\
\hline Denmark & 5 \\
\hline Estonia & 4 \\
\hline Finland & 1 \\
\hline France & 43 \\
\hline Germany & 20 \\
\hline Greece & 16 \\
\hline Hungary & 3 \\
\hline Italy & 39 \\
\hline Lithuania & 1 \\
\hline Macedonia & 6 \\
\hline Malta & 1 \\
\hline Netherlands & 2 \\
\hline Norway & 2 \\
\hline Poland & 11 \\
\hline Portugal & 9 \\
\hline Romania & 6 \\
\hline Serbia & 1 \\
\hline Slovakia & 19 \\
\hline Spain & 45 \\
\hline Sweden & 4 \\
\hline Switzerland & 7 \\
\hline UK & 36 \\
\hline Total & 338 \\
\hline
\end{tabular}

depended on the rate of transfusions that the patient was receiving. Respondents said that in $46 \%$ of cases, the results of serum ferritin monitoring "always" influenced their decisions on how they treated their patients, and "sometimes" influenced this in $47 \%$ of cases.

The factors that positively influenced detection of iron overload included patients having received $>20 \mathrm{RBC}$ units (relevant trigger in $76 \%$ of respondents), introduction of serum ferritin as a standard test $(76 \%)$, and awareness of the potential risks of frequent transfusions $(75 \%)$. On a scale from 1 (not an aid at all) to 7 (a strong aid), the mean scores for these factors were 6.1, 6.1, and 6.0, respectively.

The most important reasons for not detecting iron overload were poor patient prognosis, irregular serum ferritin monitoring, and lack of awareness about guidelines and risks of iron overload (Fig. 2). Other reasons included a low priority for iron-overload screening, and perceptions about the importance of iron overload in MDS.

Respondents' opinions were polarized about whether or not regularly monitoring serum ferritin levels was a reason for not detecting iron overload. While $30 \%$ said that this "strongly prevented" or "prevented" them from detecting iron overload, a similar proportion of respondents (38\%) believed that this "did not," or "did not at all," prevent them from detecting iron overload (Fig. 2).

Treatment of iron overload with iron chelation therapy

On a scale from 1 (not important at all) to 7 (very important), respondents said that they believed that treating iron overload in transfusion-dependent patients with MDS was "very important" (score 7; 37\% of the respondents) or "important" (score 6; 31\%); the mean score was 5.9 (Fig. 3).

The survey showed that $90 \%$ of respondents prescribed iron chelation therapy to their transfusion-dependent patients with MDS. Nonetheless, not all of these patients received iron chelation therapy; out of a mean of 34 transfusion-dependent patients with MDS seen in each of the respondents' practices or institutions in an average 6month period, 13 (38\%) received iron chelation therapy.

The factor that most led respondents to initiate iron chelation therapy was patient age of 55-64 years (relevant

Table 2 Respondent characteristics

\begin{tabular}{ll}
\hline Characteristic & $n=338$ \\
\hline Mean age (years) & 44 \\
Male/female $(n(\%))$ & $183(54) / 155(46)$ \\
Primary specialty $(n(\%))$ & \\
Hematologist & $312(92)$ \\
Oncologist & $8(2)$ \\
General practitioner & $4(1)$ \\
Transfusionist & $2(0.6)$ \\
Cytogeneticist & $1(0.3)$ \\
Other physician & $11(3)$ \\
Qualification $(n$ (\%)) & \\
Fully qualified & $293(87)$ \\
In training or completing internship & $45(13)$ \\
Work place $(n$ (\%)) & \\
Teaching hospital & $199(59)$ \\
General hospital & $95(28)$ \\
Office-based, cancer center, private & $44(13)$ \\
hospital, or other & \\
Experience as a physician, mean & 18 \\
number of years & \\
$<16$ years of experience $(n(\%))$ & $159(47)$ \\
$\geq 16$ years of experience $(n$ (\%)) & $177(52)$ \\
Mean number of patients with MDS & \\
seen per month $(n)$ & \\
As a proportion of total patients seen $(\%)$ & \\
Mean number of transfusion-dependent & \\
patients with MDS seen over a & \\
6 -month period $(n)$ & \\
\hline & \\
\hline
\end{tabular}

${ }^{\mathrm{a}}$ Two (1\%) respondents did not answer this question 
Fig. 1 Subjective importance of detection of iron overload

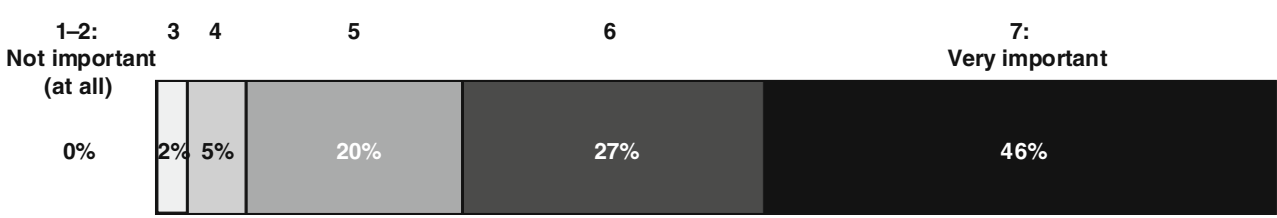

trigger in $77 \%$ of respondents). On a scale from 1 (does not lead the respondent to initiate iron chelation therapy at all) to 7 (strongly leads to initiation of iron chelation therapy), the mean score for this factor was 5.9. Other factors that most led respondents to initiate iron chelation therapy included serum ferritin levels $>1,000 \mathrm{ng} / \mathrm{mL}$ (76\%; mean score, 6.0), candidacy for allogeneic stem cell transplantation $(76 \%$; mean score, 6.0), need to prevent organ dysfunction (74\%; mean score, 6.0), $\geq 2$ RBC units transfused per month (71\%; mean score, 5.9), lifetime transfusions of $>20 \mathrm{RBC}$ units $(68 \%$; mean score, 5.8), and convenience of oral iron chelation therapy (67\%; mean score, 5.8).

The majority of respondents who prescribe iron chelation therapy $(92 \%)$ felt that a patient's serum ferritin reaching a specific level was a marker at which to initiate iron chelation therapy. The mean serum ferritin level at which these respondents initiated iron chelation therapy was $1,130 \mathrm{ng} / \mathrm{mL}$. More than half of the respondents who prescribe iron chelation therapy $(56 \%)$ said that reaching a certain number of blood units transfused was also a marker (mean of 21 units). Other markers for initiating iron chelation therapy included patients having received a particular number of transfusions (mean of 18 transfusions; $41 \%$ of respondents who prescribe iron chelation therapy) and the rate of increase of a patient's serum ferritin level (33\% of respondents who prescribe iron chelation therapy).

The strongest reasons for not initiating iron chelation therapy were mostly related to poor patient prognosis and patient age $\geq 85$ years (Fig. 4). There were polarized opinions regarding the importance of some of the reasons for not initiating iron chelation therapy. For example, while $31 \%$ of respondents replied that a high-risk MDS classification was a strong barrier in initiating iron chelation therapy, $25 \%$ thought that this did not represent a significant barrier. Similarly, while $28 \%$ said that expected noncompliance was a strong barrier in initiating iron chelation therapy, $23 \%$ thought this was a weak barrier. Some of the reasons for not initiating iron chelation therapy specifically related to the age of the patients being treated. Older patient age was less of a reason to initiate iron chelation therapy, and more of a reason not to, than younger patient age.

\section{Discussion}

This survey was undertaken to better understand what physicians perceive to be the key reasons for investigating iron overload and managing iron overload when treating

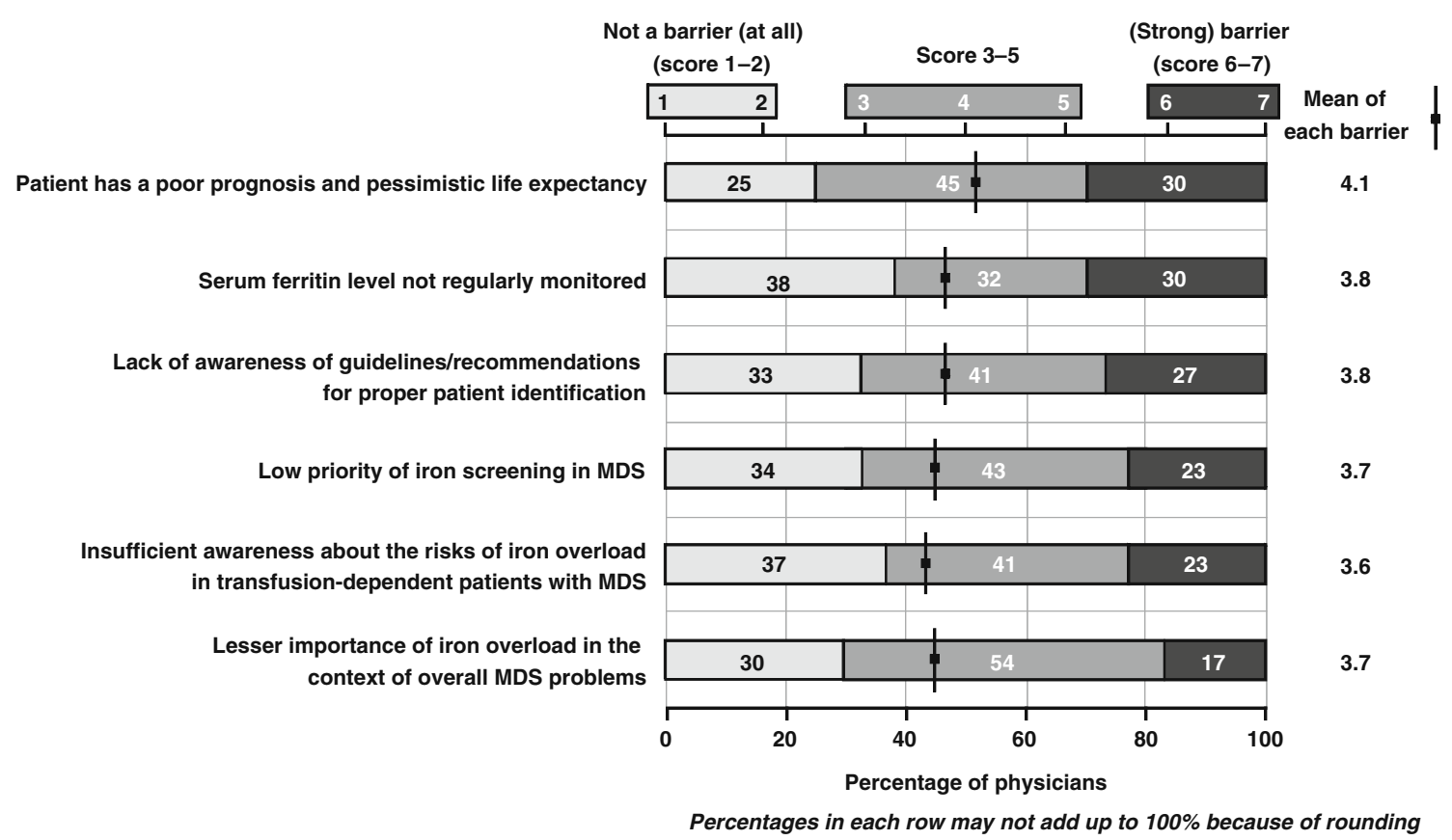

Fig. 2 The most important reservations about the detection of iron overload 
Fig. 3 Subjective importance of

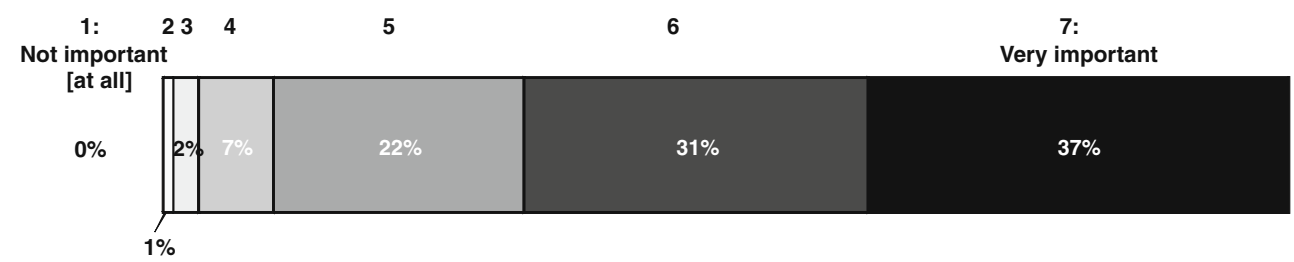

treating iron overload

transfusion-dependent patients with MDS, and the reasons that they do not. The respondents who took part in MIDIS were from multiple European countries, and the majority were experienced physicians who appeared to have a keen interest in the MDS therapy area. Over a quarter had more than 25 years of experience working as physicians, and the majority were actively involved in the diagnosis and treatment of patients with MDS. The survey has the known limitations of optional surveys; i.e., it was performed on invitation at conferences or through e-mail alerts and weblinks. Therefore, the survey may have been biased toward respondents with an interest in MDS, and who may have had a greater understanding of the management of iron overload than the general population of hematologists. Furthermore, MIDIS is not a representative sample of the hematologists from each country, and the number of respondents from each country does not accurately reflect the proportion of that country's hematologists in Europe.

Nonetheless, the results of the survey provide a valuable insight into the practices and beliefs of European physicians treating transfusion-dependent patients with MDS. MIDIS showed that approximately $70 \%$ of the respondents be- lieved that detecting and treating iron overload were important in patients with MDS. A total of $76 \%$ of physicians felt that a serum ferritin level $>1,000 \mathrm{ng} / \mathrm{mL}$ was a relevant trigger to initiate iron chelation therapy. Over $50 \%$ of the respondents monitored serum ferritin levels quarterly as a surrogate marker for iron overload in their patients, and overall, 38\% of transfusion-dependent patients with MDS were actively being treated for iron overload with iron chelation therapy. In Europe, the prevalence of MDS patients with Low/Int-1-risk MDS (according to the IPSS) is reported to be approximately $70 \%$ [2], and $39-50 \%$ of those patients are transfusiondependent [13]. Therefore, approximately one third of patients will be transfusion dependent and may be candidates for iron chelation therapy, according to published guidelines. The $38 \%$ response by the survey respondents reflects their active iron chelation treatment strategy. This is confirmed by a recent study showing that $41 \%$ of eligible patients with lower-risk MDS received iron chelation therapy in clinical practice [14]. In all, the views expressed in the survey are in line with a number of international guidelines that recommend that serum ferritin

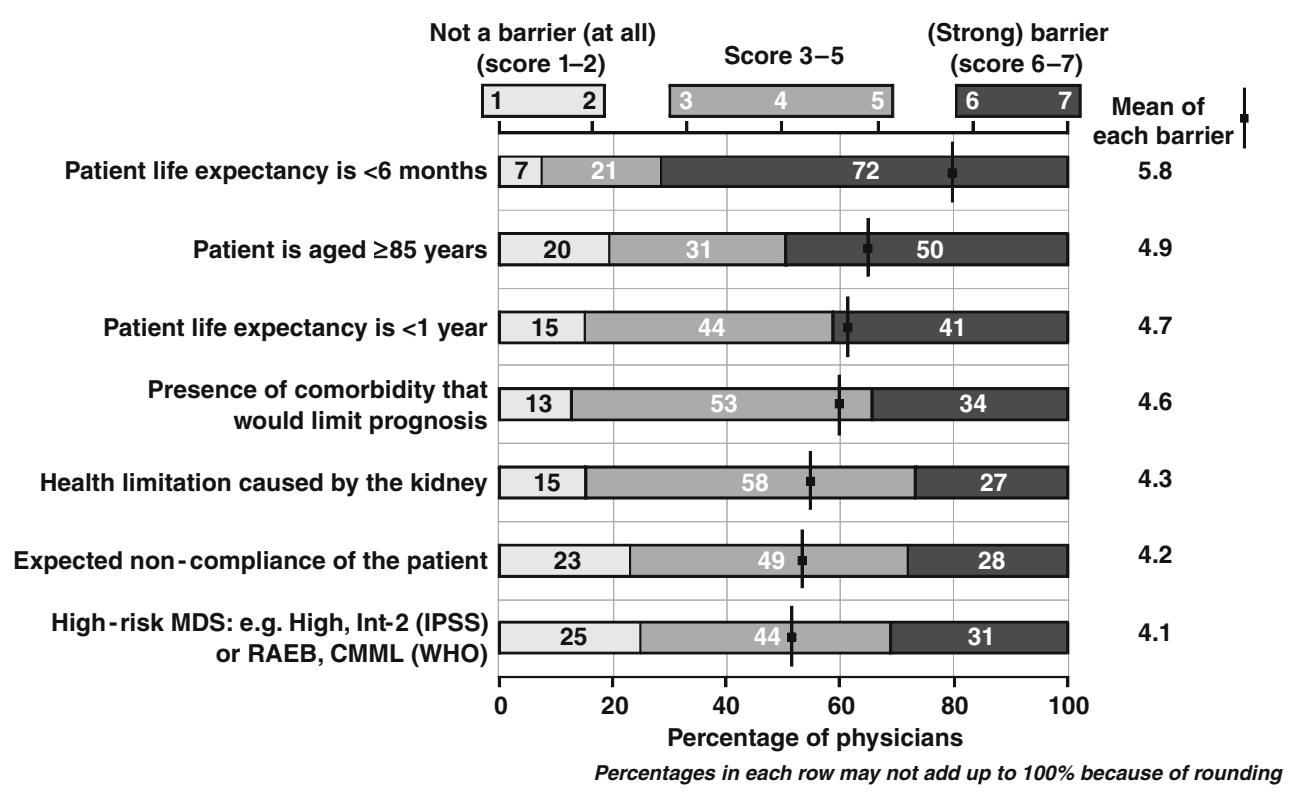

Fig. 4 The most important reservations about initiating iron chelation therapy. $R A E B$ refractory anemia with excess blasts, $C M M L$ chronic myelomonocytic leukemia, WHO World Health Organization 
levels should be assessed three to four times per year in transfusion-dependent patients with MDS, and that iron chelation therapy should be initiated when their serum ferritin level reaches $1,000 \mathrm{ng} / \mathrm{mL}$, depending on the transfusion rate [4, 15-17]. Interestingly, fewer than a quarter of the respondents considered concerns about potential reductions in patient quality of life, the cost of iron chelation therapy, and possible side effects of therapy as strong reasons for not initiating treatment. Only $23 \%$, $19 \%$, and $11 \%$ of respondents, respectively, reported these as barriers or strong barriers in initiating treatment. Furthermore, the issue of potentially reduced quality of life was polarized; the proportion of respondents who considered this to be a barrier was similar to the proportion of those who did not (23\% and $26 \%$, respectively).

Participating physicians were asked whether considering iron overload to be a lesser problem in patients with MDS was a barrier in detecting iron overload. While $17 \%$ thought this was a barrier or strong barrier, 30\% were strongly opposed to that view. This reflects the need for evidencebased data regarding the role of iron chelation in MDS. Serum ferritin is a well-known acute-phase reactant that might overestimate the iron load in the presence of other diseases, in particular infectious episodes [18]. More accurate measurements of iron overload include liver and heart magnetic resonance imaging; however, these are timeconsuming and not available at many centers. This may be one reason why one third of MIDIS respondents said that they believed that irregular monitoring of serum ferritin levels was a strong barrier in detecting iron overload, while $38 \%$ thought that this did not prevent detection.

The survey respondents were reluctant to administer iron chelation therapy to patients aged $\geq 85$ years. It is difficult to assess how transfusion dependence affects the overall survival of this elderly patient population with Low- or Int1-risk MDS. Nonetheless, it is interesting to note that the proportion of physicians opting against iron chelation therapy for this population was higher than that of physicians opting against treatment for patients with a life expectancy of less than 1 year (Fig. 4). Instead, patient age of 55-64 years was a strong reason for initiating iron chelation therapy.

In summary, this European survey has demonstrated that physicians believe that iron overload is a relevant clinical issue in transfusion-dependent patients with MDS that is worthy of investigation and treatment. Concerns over age and patient prognosis are key factors in the treatment decisions taken by physicians. An ongoing, randomized phase III trial in patients with Low- and Int-1-risk MDS on iron overload in MDS will help to clarify the role of iron chelation therapy in this patient population.

Acknowledgments This study was sponsored by Novartis Farma SpA. Financial support for medical editorial assistance was provided by Novartis Farma SpA. We thank Kathy Heptinstall, Didi Jasmin, The MDS Foundation, and the European School of Haematology for their assistance and support with MIDIS and the manuscript. We thank Roy Mazucco for medical editorial assistance with this manuscript.

Conflicts of interest AG reports receiving consultancy and speaker bureau fees and is a member of the board of directors or advisory committee for Novartis Pharma AG, Amgen Inc, GlaxoSmithKline plc, Johnson \& Johnson Services Inc, and Celgene Corp. DJ is employed by the European School of Haematology, which receives unrestricted educational grants from Novartis Pharma AG. SLdP is Head of Patient Advocacy of Novartis Oncology Region Europe and has equity in Novartis Pharma AG. SI is a full-time employee of GfK SE, the market research agency that was commissioned and paid by Novartis Farma SpA to collect and analyze the MIDIS survey data. PF reports receiving honoraria and research funding from Celgene Corp, F Hoffmann-La Roche Ltd, Ortho Biotech Products, Amgen Inc, Cephalon Inc, Merck \& Co Inc, and Novartis Pharma AG.

Open Access This article is distributed under the terms of the Creative Commons Attribution Noncommercial License which permits any noncommercial use, distribution, and reproduction in any medium, provided the original author(s) and source are credited.

\section{References}

1. Hellström-Lindberg E (2005) Management of anemia associated with myelodysplastic syndrome. Semin Hematol 42:S10-S13

2. Malcovati L, Della Porta MG, Pascutto C, Invernizzi R, Boni M, Travaglino E, Passamonti F, Arcaini L, Maffioli M, Bernasconi P, Lazzarino M, Cazzola M (2005) Prognostic factors and life expectancy in myelodysplastic syndromes classified according to WHO criteria: a basis for clinical decision making. J Clin Oncol 23:7594-7603

3. Sanz G, Nomdedeu B, Such E, Bernal T, Belkaid M, Ardanaz T, Marco V, Pedro C, Ramos F, Consuelo del Cañizo M, Luño E, Cobo F et al. (2008) Independent impact of iron overload and transfusion dependency on survival and leukemic evolution in patients with myelodysplastic syndrome. Blood 112(11):abst 640

4. Bennett JM (2008) Consensus statement on iron overload in myelodysplastic syndromes. Am J Hematol 83:858-861

5. Arrizabalaga B, del Cañizo C, Remacha A, Sanz G, Villegas A (2008) Guía clínica de quelación del paciente con síndrome mielodisplásico [Clinical guide to chelation therapy for patients with myelodysplastic syndrome (Spanish Guidelines)]. Haematologica 93(Suppl 1):3-10

6. Santini V, Alessandrino PE, Angelucci E, Barosi G, Billio A, Di Maio M, Finelli C, Locatelli F, Marchetti M, Morra E, Musto P, Visani G et al. (2010) Clinical management of myelodysplastic syndromes: update of SIE, SIES, GITMO practice guidelines. Leuk Res 34:1576-1588

7. Bowen D, Culligan D, Jowitt S, Kelsey S, Mufti G, Oscier D, Parker J (2003) Guidelines for the diagnosis and therapy of adult myelodysplastic syndromes. Br J Haematol 120:187-200

8. National Comprehensive Cancer Network. NCCN Clinical practice guidelines in oncology v.2: myelodysplastic syndromes. (2010). Available at: http://www.ncen.org/professionals/physician_gls/ $\mathrm{pdf} / \mathrm{mds}$.pdf

9. DeLoughery TG (2009) Iron: the fifth horseman of the apocalypse? Am J Hematol 84:263-264 
10. Tefferi A, Stone RM (2009) Iron chelation therapy in myelodysplastic syndrome - Cui bono? Leukemia 23:1373

11. Leukemia Research Fund. Myelodysplastic syndromes: information and education. (2010). Available at: http://www.lrf.org.uk/

12. Pullarkat V (2009) Objectives of iron chelation therapy in myelodysplastic syndromes: more than meets the eye? Blood 114:5251-5255

13. Brechignac S, Hellstrom-Lindberg E, Bowen DT, DeWitte TM, Cazzola M, Fenaux P (2004) Quality of life and economic impact of red blood cell (RBC) transfusions on patients with myelodysplastic syndromes (MDS). Blood 104(11):abst 4716

14. Raptis A, Duh MS, Wang S-T, Dial E, Fanourgiakis I, Fortner B, Paley C, Mody-Patel N, Corral M, Scott J (2010) Treatment of transfusional iron overload in patients with myelodysplastic syndrome or severe anemia: data from multicenter clinical practices. Transfusion 50:190-199
15. Suzuki T, Tomonaga M, Miyazaki Y, Nakao S, Ohyashiki K, Matsumura I, Kohgo Y, Niitsu Y, Kojima S, Ozawa K (2008) Japanese epidemiological survey with consensus statement on Japanese guidelines for treatment of iron overload in bone marrow failure syndromes. Int J Hematol 88:30-35

16. Wells RA, Leber B, Buckstein R, Lipton JH, Hasegawa W, Grewal K, Yee K, Olney HJ, Larratt L, Vickars L, Tinmouth A (2008) Iron overload in myelodysplastic syndromes: a Canadian consensus guideline. Leuk Res 32:1338-1353

17. Gattermann N, Porter J, Lopes LF, Seymour J (2005) Consensus statement on iron overload in myelodysplastic syndromes. Hematol Oncol Clin North Am 19(Suppl 1):18-25

18. Jabbour E, Garcia-Manero G, Taher A, Kantarjian HM (2009) Managing iron overload in patients with myelodysplastic syndromes with oral deferasirox therapy. Oncologist 14:489-496 